\title{
Ruptured Pulmonary Hydatid Cyst With Anaphylactic Shock and Pneumothorax
}

\author{
Mohammad Shameem MD, Jamal Akhtar MD, Rakesh Bhargava MD, Zuber Ahmed MD, \\ Nafees Ahmad Khan, and Ummul Baneen MD
}

\begin{abstract}
Hydatid cyst is a disease caused by a parasitic tapeworm, Echinococcus granulosus, and most commonly involves liver and lung. Ruptured pulmonary hydatid cyst can present a diagnostic challenge, and radiograph can be inconclusive. Anaphylactic reaction is a rare complication of ruptured pulmonary hydatid cyst. A 22-year-old male came to our emergency department in shock with symptoms of shortness of breath and altered mental status from the previous day. Radiograph showed a thin-walled circular translucent area in the right upper lung field, which was misdiagnosed as pneumothorax, and an intercostal chest tube was inserted. After 5 days, repeat radiograph revealed a cavity with an air/fluid level. The chest tube was removed and contrast-enhanced computed tomogram showed a cavity with water-lily sign, which suggests ruptured hydatid cyst. Immunoglobin-G enzyme-linked immunosorbent assay for Echinococcus was positive. The patient responded well to treatment with crystalloid infusion, supplemental oxygen, and albendazole, and then underwent surgery. Anaphylactic reaction due to rupture of a hydatid cyst is rare, but hydatid disease should be suspected in patients from areas where Echinococcus is endemic. Key words: Echinococcus; pulmonary hydatid cyst; anaphylactic shock; pneumothorax. [Respir Care 2011;56(6): 863-865. (c) 2011 Daedalus Enterprises]
\end{abstract}

\section{Introduction}

Hydatid disease is created by the larval stage of the parasitic tapeworm Echinococcus granulosus. The disease state is characterized by cyst formation in various organs. Hydatid disease frequently occurs in endemic areas, including several Mediterranean countries, New Zealand, Australia, North America, South America, Central America, and Asia. ${ }^{1}$ The liver is the most commonly affected organ, followed by the lungs. The initial growth of a cyst is usually asymptomatic, until symptoms are caused by the

\footnotetext{
All authors are affiliated with the Department of Tuberculosis and Chest Diseases, Jawaharlal Nehru Medical College, Aligarh Muslim University, Uttar Pradesh, India.

The authors have disclosed no conflicts of interest.

Correspondence: Jamal Akhtar MD, Department of Tuberculosis and Chest Diseases, Jawaharlal Nehru Medical College, Aligarh Muslim University, Aligarh 202002, Uttar Pradesh India. E-mail: akhtar.jamal10@ gmail.com.
}

DOI: $10.4187 /$ respcare. 00821 cyst's space-occupying mass effect, mechanical obstruction, rupture, or secondary allergic reaction. Anaphylactic reactions are rare presentations of ruptured pulmonary hydatid cyst.2,3 Diagnosis of a ruptured hydatid cyst is difficult and hydatid cyst may be misdiagnosed as another lung disease, which delays correct treatment. We report a case of spontaneous pulmonary hydatid cyst rupture that was first misdiagnosed as an anaphylactic reaction and then misdiagnosed and mismanaged as a pneumothorax. The patient gave written informed consent for us to publish this case.

\section{Case Report}

A 22-year-old male presented to the emergency department with complaints of shortness of breath and altered mental status from the preceding day. There was no history of trauma, fever, cough, chest pain, or hemoptysis. His blood pressure was $70 / 50 \mathrm{~mm} \mathrm{Hg}$, heart rate 124 beats/ min, respiratory rate 40 breaths/min, and temperature $38.3^{\circ} \mathrm{C}$. He was cyanotic and his oxygen saturation was $77 \%$. His respiratory pattern was shallow. Air entry was decreased anterior on his right side in the supramammary 


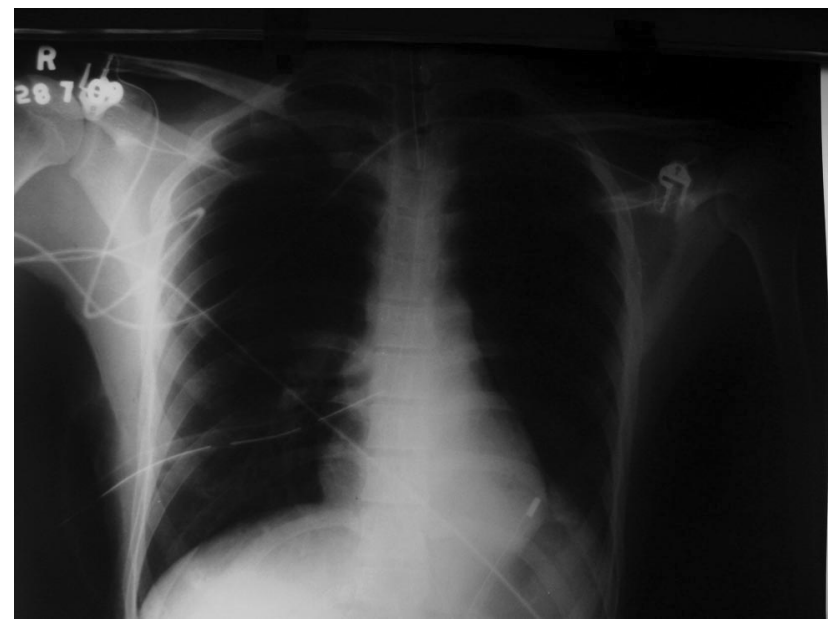

Fig. 1. Radiograph shows a thin-walled translucent area in the right upper lung field, which was misdiagnosed as pneumothorax, and an intercostal chest tube.

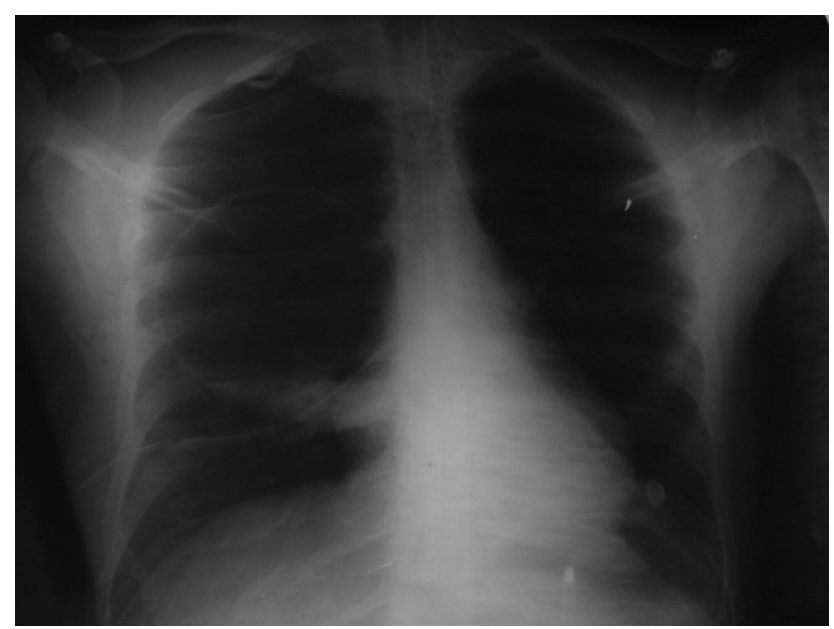

Fig. 2. Radiograph shows a cavity with an air/fluid level, and an intercostal chest tube.

area and posterior in his suprascapular and interscapular area, and there were bilateral rhonchi. He was agitated and not oriented to person, place, or time. He underwent fluid resuscitation with crystalloid infusion. He received supplemental oxygen, subcutaneous adrenaline, and intravenous hydrocortisone, after which his blood pressure, respiration, and mental status improved.

Radiograph showed a thin-walled circular translucent area in the right upper lung field (Fig. 1). A misdiagnosis of pneumothorax was made, and an intercostal chest tube was placed. After 5 hospital days, a repeat radiograph revealed a cavity with an air/fluid level, and the chest tube in situ (Fig. 2). The chest tube was then removed and a contrast-enhanced computed tomogram showed a cavity with water-lily sign, which suggested of a ruptured hydatid cyst (Fig. 3). Immunoglobin-G enzyme-linked immunosor-

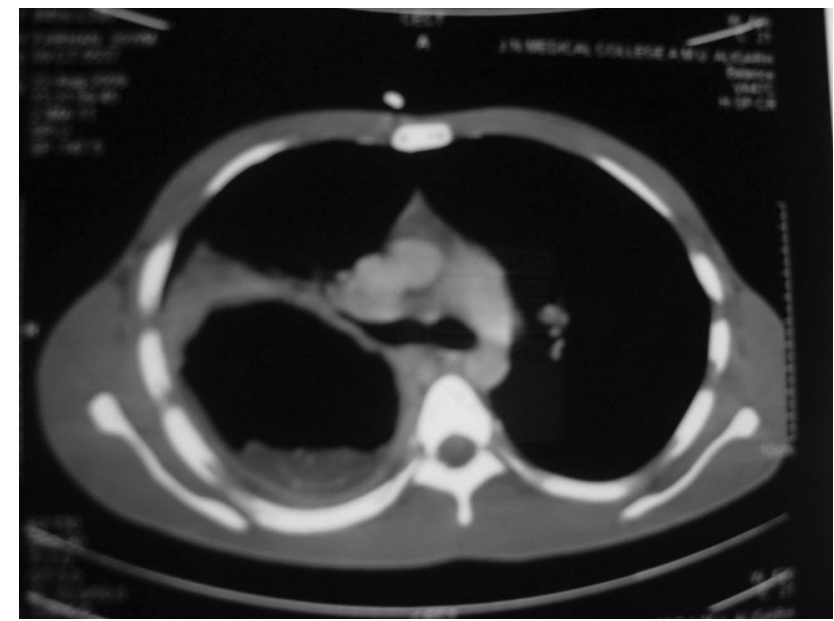

Fig. 3. Computed tomogram shows a cavity with water-lily sign, which suggests ruptured hydatid cyst.

bent assay for Echinococcus was positive, and the revised diagnosis was spontaneously ruptured hydatid cyst with anaphylactic shock and respiratory distress.

Per standard medical treatment for a ruptured hydatid cyst, we administered oral albendazole, $400 \mathrm{mg}$, twice daily. ${ }^{4} \mathrm{He}$ responded well to albendazole and supportive care. After 21 days he underwent surgery for the ruptured cyst, and histopathology confirmed hydatid cyst. His postsurgery recovery was uneventful, and he has had no longterm complications.

\section{Discussion}

Pulmonary hydatid cyst most commonly appears in the lower lobe of the right lung, and is usually solitary. These cysts may initially be asymptomatic, and may be diagnosed incidentally during a radiological evaluation for other reasons. On the other hand, a hydatid cyst may present with symptoms, such as chest pain, hemoptysis, dyspnea, and fever. Kuzucu et $\mathrm{al}^{5}$ reported chest pain (50\%), cough (27\%), dyspnea (18\%), and fever (12\%) as the most common presenting symptoms in patients with intact pulmonary hydatid cysts. However, $27 \%$ of patients were asymptomatic. Pulmonary hydatid cysts rupture in about a third of patients, ${ }^{6}$ releasing a highly antigenic fluid into the bronchus, which can cause secondary hydatid spread, asphyxia, anaphylactic shock, acute respiratory failure, massive hemoptysis, and circulatory collapse. Ruptured pulmonary hydatid cysts generally present with chest pain (49\%), cough (46\%), dyspnea (42\%), hemoptysis (33\%), fever $(36 \%)$, and sputum production (33\%), whereas only $3 \%$ of patients with ruptured pulmonary hydatid cyst are asymptomatic. ${ }^{5}$ Anaphylactic shock is a rare reaction to a ruptured pulmonary hydatid cyst.2.,3 In our patient, shock and respiratory distress were present, but other diagnostic 


\section{Ruptured Pulmonary Hydatid Cyst With Anaphylactic Shock and Pneumothorax}

symptoms such as rash and edema were absent. Fanne et al reported a similar case of ruptured pulmonary hydatid cyst with shock and respiratory distress but absence of rash and edema. $^{2}$

Ruptured pulmonary hydatid cyst is often difficult to diagnose because after rupture into a bronchial lumen, radiographic appearance can be widely varied. It may be misdiagnosed as pneumonia, tuberculosis, lung abscess, tumor, or pneumothorax. ${ }^{7}$ Poyrazoglu et al reported ${ }^{8}$ a case of ruptured pulmonary hydatid cyst that was diagnosed as a lung mass. Air/fluid level, air bubbles, and/or cavitation may also be seen in a ruptured cyst, ${ }^{9}$ mimicking a lung abscess or tubercular cavity. The edges of the fluidfilled cyst may be blurred, mimicking inflammation or pneumonia. In some cases, when all the contents of the cyst are released and the cyst is almost completely filled with air, a thin-walled hollow air-filled cavity or tension air bag ${ }^{6}$ can be diagnosed as pneumothorax. This appearance in our patient led to the initial diagnosis of pneumothorax.

The parasitic membrane may partially detach from the adventitia, drop, and float in the residual fluid. The endocyst membrane floating on top of the remaining fluid is called the water-lily sign, a highly characteristic radiographic image for hydatid cyst rupture. Radiography remains the initial imaging modality for detecting hydatid cysts. Ultrasonography, computed tomography, and magnetic resonance imaging can also be used. Computed tomography has a sensitivity of $100 \%$ in identifying cyst rupture. In the study by Erdem et al, ${ }^{10}$ plain chest radiograph detected only 57 of 79 pulmonary cysts detected via computed tomogram.

The treatment of ruptured pulmonary hydatid cysts is principally surgical. Albendazole is given as a complement to surgical treatment, to avoid recurrence, ${ }^{11}$ and in patients who cannot undergo surgery. ${ }^{12}$ Albendazole is particularly effective on smaller cysts. ${ }^{11}$ Treatment of the other symptoms is standard: adrenaline for anaphylactic shock; $100 \%$ oxygen; and colloid or crystalloid to replenish intravascular volume.

This case should raise awareness of an uncommon presentation of hydatid disease in medical departments, where most patients with this type of presentation would be first seen, especially in regions with limited surgical resources. This case should also raise awareness of hydatid disease worldwide; with global travel and immigration, patients with hydatid disease may present to physicians who are unfamiliar with it varied radiologic features. Hydatid cyst rupture should be suspected in patients from areas where Echinococcus is endemic.

\section{ACKNOWLEDGMENTS}

We thank Belinda Peace MD for editorial assistance.

\section{REFERENCES}

1. Bchir A, Hamdi A, Jemni L, Dazza MC, Allegue M, Braham MS, et al. Serological screening for hydatidosis in households of surgical cases in central Tunisia. Ann Trop Med Parasitol 1988;82(3):271273.

2. Fanne RA, Khamaisi M, Mevorach D, Leitersdorf E, Berkman N, Laxer U, et al. Spontaneous rupture of lung echinococcal cyst causing anaphylactic shock and respiratory distress syndrome. Thorax 2006;61(6):550.

3. Mooraki A, Rahbar MH, Bastani B. Spontaneous systemic anaphylaxis as an unusual presentation of hydatid cyst: report of two cases. Am J Trop Med Hyg 1996;55(3):302-303.

4. Keshmiri M, Baharvahdat H, Fattahi SH, Davachi B, Dabiri RH, Baradaran $\mathrm{H}$, et al. A placebo controlled study of albendazole in the treatment of pulmonary echinococcosis. Eur Respir J 1999;14(3): 503-507.

5. Kuzucu A, Soysal O, Ozgel M, Yologlu S. Complicated hydatid cysts of the lung: clinical and therapeutic issues. Ann Thorac Surg 2004;77(4):1200-1204.

6. Aytac A, Yurdakul Y, Ikizler C, Olga R, Saylam A. Pulmonary hydatid disease: report of 100 patients. Ann Thorac Surg 1977;23(2): 145-151.

7. Zhang Q, Huang TM, Li BZ, Li ZL, Liao KX. [Misdiagnosis of pulmonary hydatid cyst rupture: report of 38 cases]. Zhonghua Jie $\mathrm{He} \mathrm{He} \mathrm{Hu}$ Xi Za Zhi 2003;26(8):474-476. Article in Chinese.

8. Poyrazoglu H, Tor F, Avsar MK, Bayraktar I, Ulus T. An unusual approach to a ruptured hydatid cyst case which had been treated with a wrong diagnosis. Indian J Thorac Cardiovasc Surg 2008;24(1):2223.

9. Zidi A, Ben Miled-Mrad K, Hantous-Zannad S, Fathallah B, Mestiri I, Baccouche I, et al. [Computed tomography of complicated pulmonary hydatid cyst by rupture in the bronchi]. J Radiol 2007;88(1 Pt 1):59-64. Article in French.

10. Erdem CZ, Erdem LO. Radiological characteristics of pulmonary hydatid disease in children: less common radiological appearances. Eur J Radiol 2003;45(2):123-128.

11. Rai SP, Panda BN, Ganguly D, Bharadwaj R. Pulmonary hydatid: diagnosis and response to hypertonic saline irrigation and albendazole. Med J Arm Forces India 2005;61(1):9-12.

12. Force L, Torres JM, Carrillo A, Busca J. Evaluation of eight serological tests in the diagnosis of human echinococcosis and follow-up. Clin Infect Dis 1992;15(3):473-480. 